\title{
Classification of Broken Rice Kernels using 12D Features
}

\author{
SUNDER ALI KHOWAJA*, FARZANA RAUF ABRO**, SHEERAZ MEMON***, AND PARUS KHUWAJA**** \\ RECEIVED ON 13.11.2015 ACCEPTED ON 16.02.2016
}

\begin{abstract}
Integrating the technological aspect for assessment of rice quality is very much needed for the Asian markets where rice is one of the major exports. Methods based on image analysis has been proposed for automated quality assessment by taking into account some of the textural features. These features are good at classifying when rice grains are scanned in controlled environment but it is not suitable for practical implementation. Rice grains are placed randomly on the scanner which neither maintains the uniformity in intensity regions nor the placement strategy is kept ideal thus resulting in false classification of grains. The aim of this research is to propose a method for extracting set of features which can overcome the said issues. This paper uses morphological features along-with gray level and Hough transform based features to overcome the false classification in the existing methods. RBF (Radial Basis function) is used as a classification mechanism to classify between complete grains and broken grains. Furthermore the broken grains are classified into two classes' i.e. acceptable grains and non-acceptable grains. This research also uses image enhancement technique prior to the feature extraction and classification process based on top-hat transformation. The proposed method has been simulated in MATLAB to visually analyze and validate the results.
\end{abstract}

Key Words: Rice Classification, Feature Extraction, Image Analysis, Hough Transform, Radial Basis Function.

\section{INTRODUCTION}

A gricultural Industry is referred to as one of the widespread and largest industry of the world. Due to the increasing demand of agricultural commodities the need for efficient and automated resource management for this sector is desperately escalating. Recent advancement in technology has encouraged the researchers to work on developing algorithms for automated way of quality assessment hence replacing human inspection by machine intelligence [1]. Amongst all agricultural products rice is considered to be one of the major commodities as it is the major source of food for half of the world population. Apart from this rice is also considered to be the most flexible cereal as it adapts the agro-ecological variations suggesting that it can grow under saline conditions, in flooded areas, in freshwater and in dry fields as well [2]. Various studies have also suggested that by employing machine vision algorithms high quality standards can be achieved accurately along with a cost effective approach [3].

\footnotetext{
* Institute of Information \& Communication Technology, University of Sindh, Jamshoro.

** Department of ElectronicÓ Engineering, Mehran University of Engineering \& Technology, Jamshoro.

*** Department of Computer Systems Engineering, Mehran University of Engineering \& Technology, Jamshoro.

**** Institute of Business Administration, University of Sindh, Jamshoro.
} 
The quality of rice grains can be evaluated by taking into account some parameters such as shape, size, whiteness and texture. Based on these parameters the rice kernels can be classified as whole kernels or broken kernels. This classification is considered to be an imperative characteristic to determine the quality of rice kernels. With reference to the whole kernel the rice kernel can be assumed as a broken kernel if it is three fourth of its size [4]. The quality assessment based on the number of broken kernels by manual human inspection is neither efficient (i.e. time to assess) nor reliable (i.e. accuracy). The assessment can be improved in terms of efficiency and reliability by employing machine vision techniques signifying that the images of rice kernels can be acquired by digital camera or scanners [5].

The design of such machine vision systems require set of features to be extracted from the rice kernel images to classify between complete kernels and broken rice kernels. A thorough research has been carried out to extract features that can enhance the accuracy of the system, these features include geometrical features [6], color based features [7], texture based features [8] and shape based features [9].

In addition to the feature extraction, classification process is given equal importance for efficient quality assessment. Several classification methods have been proposed in the past for identification of objects, amongst them the most popular ones are HMM (Hidden Markov Models) [10], GMM (Gaussian Mixture Models) [11], SVM (Support Vector Machines) [12], MEMM (Maximum Entropy Markov Model) [13] and ANN (Artificial Neural Networks) [14].

In this paper a rice classification method with a $12 \mathrm{D}$ feature vector has been proposed based on morphological [8], gray-level [15] and Hough transform [16] based features to accurately classify the complete rice kernels and broken rice kernels. Furthermore rice kernels will be classified as acceptable rice-kernels and non-acceptable rice kernels. Radial basis function with exact fitting neurons [17] method has been used for the classification of rice kernels which uses only one hidden layer with exact number of neurons with reference to the number of features used in feature vector. For accurately extracting features from the rice kernel images it is necessary to make the image undergo some pre-processing techniques for enhancing and making the image compatible for further processing, in this regard morphological image processing i.e. top hat transformation [18] has been used for image enhancement prior to the feature extraction process. The reason for employing the proposed method is to eliminate the false rice kernels which are merged with border, hence they are perceived to be the complete rice grains because of their augmented area section and to intensify the accuracy of the classification system.

Furthermore, this paper has been divided into five sections where section 2 will deal with the existing work being carried out in the similar field followed by section 3 carrying out the comprehensive discussion on the proposed methodology. Section 4 will highlight the results being procured by implementing the proposed method and section 5 will finally present the conclusion and discussion based on the acquired results.

\section{RELATED WORK}

During the last decade several research has been carried out regarding the classification of rice kernels, most of the research is based on particular varieties of rice but are not generalized in a way that the same method could be applied to other varieties of rice in an efficient manner. Moreover there is a very limited literature available for study being carried out on the classification of complete rice kernels and broken rice kernels which is a basis to make the classification method generalized. However some of the literature work regarding classification of varieties and classification of broken rice is consolidated in this section. 
Sapirstein, et. al. [19] first proposed the classification method for classifying grains such as rye, barley, oats and wheat by employing shape and size parameters using linear discriminant analysis. Zayas, et. al. [20] proposed a method for classifying wheat and non-wheat class suggesting that the non-wheat class consists of weed seeds and stones. Multivariate discriminant analysis was used to identify the separable classes but physical separation of two classes i.e. wheat and non-wheat grouping was required a prior of this classification. Ding and Gunasekaran [9] proposed a method for extracting features based on statistical model based feature extractor along with back propagation neural network and minimum intermediate zone classifiers for classification of corn kernels. Zayas, et. al. [21] again proposed a classification mechanism for identifying wheat kernels based on morphometrical and hardness parameters fetched from force-deformation curves to characterize wheat from seven different varieties. Wee, et. al. [22] proposed a classification method for rice kernels based on Zernike moments based features with Fuzzy ARTMAP i.e. multidimensional maps neural networks, in order to reduce the learning time and improve the computation speed so that the said system could be implemented in real time. Danying, et. al. [23] proposed a mechanism for classifying rice in of the 3 categories i.e. high, middle and low, suggesting that high quality refers to head rice whereas middle and low quality refers to chalkiness and amylose content. Correlation technique was used to classify rice kernels in one of the three proposed categories. Liu, et. al. [14] proposed an algorithm for rice kernel classification based on color and morphological features, neural networks along with discriminant analysis were used to classify rice kernels for six different varieties. Wee, et. al. [24] proposed sorting algorithm for rice kernels based on Zernike moments and neural networks suggesting that is q-recursive method is used to compute the Zernike moments the overall classification time can be reduced by $25 \%$ hence making the classification system fast and less computationally complex. Agustin and Byung-Joo [25] proposed an evaluation framework for determining the quantity of brewers, broken rice and head rice using by using geometric and color based features. Probabilistic neural network classifier is used to characterize defects in rice kernels. Tom Pearson [26] proposed an FPGA system integrated with CMOS based color image sensor to separate white and red rice kernels, and to remove pop-corn by taking into account the blue-eye damage indicated by blue discoloration. Pabamalie and Premaratne [27] proposed the extraction of thirty one texture and color features to classify rice kernels for 4 different varieties. BPNN (Back Propagation Neural Network) was used as a classifier to perform the sorting of rice kernels. Rad, et. al. [8] proposed the extraction of twenty two features based on color and texture for classifying four varieties of rice kernels using BPNN as the classifier. Rad, et. al. [28] again proposed the classification mechanism of rice kernels for 5 different varieties but this time they considered 18 features based on morphological structures, same classifier i.e. BPNN was employed to perform the classification of rice kernels. Kaur and Singh [12] proposed the extraction of ten geometric features to classify the defective or broken rice kernels and complete rice kernels. They used maximum variance method to segment out the kernels from the background and multi-class SVM to classify the complete rice kernels and broken rice kernels. Pazoki, et. al. [29] proposed the classification of rice kernels amongst 5 different varieties by taking into account 39 features based on color, morphology and shape in accordance with MLP (Multilayer Perceptron) and Neuro-fuzzy classifier working in a cascaded approach to categorize the rice kernels. Xu, et. al. [30] proposed an improved version of PCA (Principle Component Analysis) to extract features from 5 varieties of rice kernels i.e. regular PCA combined with Wilks distribution. Probabilistic Neural Network was used to classify the rice kernels based on the features extracted. Abdullah, et. al. [31] suggested a 
method based on PCA and Linear Discriminant Analysis along with K-Nearest Neighbor to classify the Aromatic rice kernels from other varieties of rice. LOO (Leave-OneOut) validation approach was applied to corroborate the results fetched from the proposed approach. Wang, et. al. [32] proposed that the morphological features to be extracted from hyperspectral images of rice kernels followed by the dimensionality reduction using PCA and finally the classification is performed by employing BPNN. They analyzed seven optimal wavelengths on spectral data to extract the desired features.

The consolidated literature work mostly represent the work for classifying the specific rice kernels amongst different varieties of rice however, a very few paper actually take into account the actual quality assessment parameters i.e. the classification of complete and broken rice kernels within a specific variety of rice kernels. Most of the papers in this section use more than 15 features which increases the computational complexity of the classification system but in the proposed approach we are using 12 features for classification hence making the system less computationally complex.

\section{PROPOSED METHODOLOGY}

This paper presents a new approach for grading rice kernels into two main categories which is complete rice kernels and broken rice kernels and further the grading will be applied to the later category for identifying the acceptable kernels and non-acceptable kernels. For accomplishing the said task 12D feature vector has been extracted based on morphological, gray level and Hough transform based features. The phases of ensuing process for execution of kernel grading operation is identified as: (1) Pre-processing for enhancement of rice kernel images which will be accomplished by gray-level homogenization (2) Extraction of features from homogenized image (3) Classifying the pixels which are complete and acceptable rice kernels.

\subsection{Pre-Processing}

Certain constraints affect the feature extraction process of rice kernel images which include noise and poor contrast, to overcome these limitations two processes will be applied to make the image suitable for feature extraction process. The said processes are Background homogenization and image enhancement.

Background homogenization process is applied to cop up with the intensity variations in the image, assuming that the acquisition device may introduce noise or changes in lighting conditions while image acquisition. Therefore, it is necessary to apply intensity homogeneity as the feature extraction process takes into account the gray-level based features. In order to ensure the homogenization, a large arithmetic mean is applied to obtain the shade-corrected image. A convolution operation of $11 \times 11$ Gaussian mask having mean 0 and standard deviation 0.86 with the acquired image is performed followed by a $67 \times 67$ mean filter which is applied to the resultant image to produce a background image with average gray level pixels only. An iterative process of image subtraction will be applied for calculating the difference of each pixel from the filtered image with reference to the original acquired image. Mathematical representation of the Gaussian kernel and image subtraction is given in Equations (1-2). The reason for applying a mean filter of $67 \times 67$ size is to avoid the strong bias on the FOV (Field-of-View) near borders by the dark external regions, for eliminating this problem the said mean filter is used to replace the average gray values with out of the FOV gray values. The use of averaging filter followed by the subtraction process as shown in Equation (2) is already been proposed in number of studies [36-38].

$$
\begin{aligned}
& G K_{\mu, \sigma}^{n}=G K_{0,0.86}^{11} \\
& I_{E}=I_{O}-I_{F}
\end{aligned}
$$


Where $I_{E}, I_{O}$ and $I_{F}$ refers to enhanced image, original image and filtered image respectively. In literature image subtraction and image division has been extensively used to shade-correction and studies also reveal that both these approached yield the same result [38] therefore in our approached we have employed image subtraction process for the said purpose. The shade corrected image is then homogenized so that the image could cover whole range of gray values. The transformation is represented in Equations (3-4) for the same.

$$
I_{\text {но }}=\left\{\begin{array}{cc}
255 & \text { if } I_{E}>255 \\
0 & \text { if } I_{E}<0 \\
I_{e q} & \text { Otherwise }
\end{array}\right.
$$

Where

$$
I_{e q}=I_{E}-\max \left(I_{E}\right)+128
$$

Where $\mathrm{I}_{\mathrm{HO}}$ is the homogenized image and $\mathrm{I}_{\mathrm{eq}}$ is the equalized image covering the complete range of gray values. This homogenized image will then be enhanced by applying morphological opening operation using a structuring element of radius 8 (also known as the top hat transformation) on the complemented homogenized image as represented in Equation (5).

$$
I_{T}=I_{H O}^{\prime}-\text { Image Opening }\left(I^{\prime}{ }_{H O}\right)
$$

\subsection{Feature Extraction}

This stage aims to characterize the rice kernels using the feature vector in such a way that they can be used to categorize the rice kernels in one of the three classes' i.e. complete rice kernels, broken but acceptable rice kernel and broken and unacceptable rice kernels. Following are the set of features which are used to categorize the rice kernels in one of three categories.

\subsubsection{Morphological Features}

Average Length is considered to be the simplest feature which determines the absolute length of each rice kernel by measuring the Euclidean distance between the most distant points. In our case the length refers to the Euclidean distance between the major axis length and the minor axis length of the rice kernel and $\mathrm{N}$ is total number of rice kernels in the image, the feature of average length can be represented as in Equation (6).

$$
f_{1}=\frac{\sum \| \text { Major Axis Length }- \text { Minor Axis Length } \|}{N}
$$

Aspect Ratio Diameter which is a ratio of shortest to the longest diameters as represented in Equation (7).

$\mathrm{f}_{2}=\frac{\mathrm{d}_{\text {minimum }}}{\mathrm{d}_{\text {maximum }}}$

Shape Feature is defined as the ratio of root mean square deviation to average mean of the distances and is represented in Equation (8).

$\mathrm{f}_{3}=\frac{\mathrm{d}_{\mathrm{rms}}}{\mathrm{d}_{\mathrm{avg}}}$

Where the mathematical representation of $\mathrm{d}_{\mathrm{rms}}$ is represented in Equation (9).

$\mathrm{d}_{\mathrm{rms}}=\sqrt{\frac{\left(\mathrm{d}_{\text {maximum }}-\mathrm{d}_{\mathrm{avg}}\right)^{2}+\left(\mathrm{d}_{\mathrm{avg}}-\mathrm{d}_{\text {minimum }}\right)^{2}}{2}}$

Compactness Ratio determine the shapes with elongated feature and takes into consideration the ratio of area to perimeter length of the rice kernel. This feature is responsible for scale variance and diagonal perimeter connectivity. The ratio can be expressed as in Equation (10).

$$
f_{4}=4 \pi \frac{A}{P^{2}}
$$

Region Diameter determine the regions having area with the same diameters and its mathematical expression is given in Equation (11). 


$$
f_{5}=\sqrt{\frac{4 A}{\pi}}
$$

Solidity Feature determines the proportion of the pixels. This feature is used to overcome the problem related to merger of rice kernels as shown in Fig. 1. The two rice kernels which are merged and considered to be single rice kernel can be unmerged using this feature with the threshold level 0.8 . This feature can be mathematically expressed as in Equation (12), though figure 1 only highlight the mergers amongst the rice kernels however, the merger of rice kernels with the boundary of the image is not shown. This feature can only be used for the mergers indicated in figure 1, another feature i.e. Hough Transform based feature is used for coping with boundary merging problem and is discussed in the later section.

$$
\mathrm{f}_{6}=\frac{A}{C_{A}}
$$

Where $\mathrm{C}_{\mathrm{A}}$ is the convex area i.e. which determines the convex hulls passing through the bounding boxes or can be said as the region inside or outside the hull.

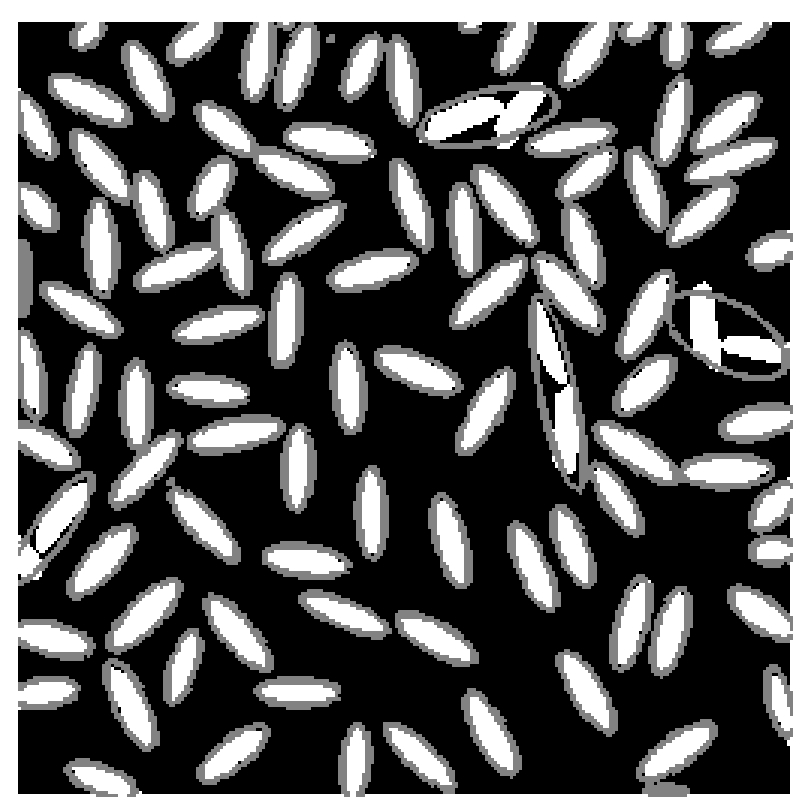

FIG. 1. MERGER OF RICE KERNELS VERY CLOSE TO EACH OTHER

\subsubsection{Gray-Level Based Features}

These features highlight the variations with respect to the neighborhood pixels suggesting that the gray-level descriptors when applied to the homogenized image considers only small set of region centered around $(a, b)$. A squared window centered around $(a, b)$ can get the set of coordinates $R_{a, b}^{w}$ where w is the size of the window. The descriptors representing gray-level features can be expressed in Equations (13-17) [15].

$$
\begin{aligned}
& \mathrm{f}_{7}(\mathrm{a}, \mathrm{b})=\mathrm{I}_{\mathrm{HO}}(\mathrm{a}, \mathrm{b}) \\
& \mathrm{f}_{8}(a, b)=\operatorname{std}{ }_{(a, b) \in R_{a, b}^{11}}\left\{I_{H O}(a, b)\right\} \\
& \mathrm{f}_{9}(a, b)=I_{\mathrm{HO}}(a, b)-\operatorname{mean}_{(a, b) \in R_{a, b}^{11}}\left\{I_{H O}(a, b)\right\} \\
& \mathrm{f}_{10}(a, b)=\max _{(a, b) \in R_{a, b}^{11}}\left\{I_{\mathrm{HO}}(a, b)\right\}-I_{\mathrm{HO}}(a, b) \\
& \mathrm{f}_{11}(a, b)=I_{H O}(a, b)-\min _{(a, b) \in R_{a, b}^{11}}\left\{I_{H O}(a, b)\right\}
\end{aligned}
$$

\subsubsection{Hough Transform Based Features}

This feature employs a voting process of peaks to find the elliptical structures with in a window and to determine whether the formation of elliptical structure is genuine or influenced by any noise in the said window. The motive of the employment of this technique is as the noise level increases votes will also increase till the saturation region occurs where the presence of an elliptical structure dominates the votes to the noise level. Once this level is attained the points are stored as a feature to differentiate between the presence of an elliptical structure and the similar structure generated by the noise level. The last feature can be expressed mathematically as shown in Equation (18).

$$
P_{C}=\frac{V_{n}-\bar{V}_{A}}{\sigma}
$$


Where $\mathrm{P}_{\mathrm{C}}$ is the peak confidence measure, $\mathrm{V}_{\mathrm{n}}$ is the number of Hough peaks and $\bar{V}_{A}$ is the mean of Hough peaks excluding the peak itself and $\sigma$ is the standard deviation of peaks. The mathematical representation of these parameters are shown in Equations (19-21).

$V_{n}=\max V_{M}, \forall M \in 1, \ldots, 11$

$\bar{V}_{A}=\frac{1}{n} \sum_{M=1}^{n} V_{M}, M \neq \max V$

$\sigma^{2}=\frac{1}{n} \sum_{M=1}^{n}\left(V_{M}-\bar{V}_{A}\right)^{2}, M \neq \max V$

The feature will take into account the peak confidence measure suggesting that if the peak confidence is less than the threshold level within the window then it is assumed to be the impact of noise level and the feature will return 0 , similarly if the peak confidence is greater than the threshold level within the window it will be considered as 1 which refers to the presence of elliptical shape. The threshold is selected as 0.9 as per the experimental tests conducted. By experimental results it is meant that the other values i.e. less than 0.87 and greater than 0.93 were not providing desired results. However, the results in the said range were all yielding the same result therefore threshold value of 0.9 is opted for generalizing this feature. The mathematical representation of the last feature is expressed in Equation (22).

$$
f_{12}= \begin{cases}1, & \text { if } P_{C}>T \\ 0, & \text { Otherwise }\end{cases}
$$

\subsection{Classification Stage}

Each object detected from the rice kernel images will be represented in 12D feature space as shown in Equation (23).

$F(a, b)=\left(f_{1}(a, b), f_{2}(a, b), \ldots f_{12}(a, b)\right)$
The classifier used in the proposed approach is exact fitting version of RBF suggesting that the exact fitting method consists of only one hidden and output layer based on the neurons equivalent to the defined features, in our case 12 neurons will be used for the classification purpose. The training set for the said classifier is expressed in Equation (24).

$$
T_{D 1}=\left\{\left(F^{(12)}, C_{P}^{(12)}\right) \mid P \in\{1,2\}\right\}
$$

Where $\mathrm{T}_{\mathrm{D}}, \mathrm{F}$ and $\mathrm{C}$ refers to Training data, Feature vector and Classes. Once the training data is established the neural network is trained using OLS (Orthogonal Least Squares) method as used in [17]. When applied the classification on the rice kernel images, each rice kernel will be assigned either class 1 i.e. complete rice kernels or class 2 i.e. broken rice kernels. Further the feature vector is reduced to 9 features as expressed in Equation (25).

$F(i, j)=\left(f_{1}, f_{2}, f_{4}, f_{5}, f_{7}, \ldots f_{11}\right)$

For further classification of broken kernels into two classes i.e. class 1 for acceptable rice kernels and class 2 for non-acceptable rice kernels. Training data set based on the feature vector in Equation (25) can be expressed as in Equation (26).

$$
T_{D 2}=\left\{\left(F^{(9)}, C_{L}^{(9)}\right) L \in\{1,2\}\right\}
$$

\section{RESULTS AND DISCUSSION}

The performance of the classification system depends on the dataset used for training purpose. In this regard, images of the rice kernels are acquired by Nikon D400 camera with zoom lens of $18-25 \mathrm{~mm}$ with a fixed aperture size of 3.5, 20 images for each rice kernel i.e. complete grain, broken but acceptable kernel and broken but unacceptable kernel is acquired for training the said system, which makes total of 480 samples of rice kernels as used in $[4,12,35]$. The proposed method is tested on 
10 images containing 799 samples of rice kernels for complete kernels, broken kernels but acceptable and broken kernels but unacceptable kernels. Fig. 2 show the images used for testing. The images chosen for testing is due to the uniformity in the acquisition device and the background for all images in the testing set. The proposed method is evaluated in terms of $\mathrm{Se}$ (Sensitivity), Sp (Specificity), NPV (Negative Predicted Value), PPV (Positive Predicted Value) and Acc (Accuracy). Se and $\mathrm{Sp}$ are the ratios of complete rice kernels and broken rice kernels in tier 1 and the ratio of acceptable broken rice kernels and unacceptable broken rice kernels. Acc is the global measure of correctly classified kernels in the respective category. Table 1 show the selected thresholds for the morphological features taken into account for the proposed method to detect complete rice kernels. Table 2 show the selected thresholds for the same features considered to detect the acceptable rice kernels. A range of threshold values were taken into account for conducting experimental tests using the proposed method, based on the optimal results the threshold values were finalized as shown in

Tables 1-2.Finally Table 3 is constructed to show the performance results after applying the proposed method on rice kernel images. Figs. 3-show some of the visual results acquired when applied the proposed method on the testing dataset and Fig. 5 show the ROC curve based on the results presented in Table 3.

TABLE 1. THRESHOLD SELECTION OF MORPHOLOGICAL FEATURES FOR COMPLETE GRAIN CLASSIFICATION

\begin{tabular}{|c|c|c|}
\hline No. & Feature & Threshold Selection \\
\hline 1. & Average Length & 7.43 \\
\hline 2. & Aspect Ratio & 1.83 \\
\hline 3. & Shape feature & 0.27 \\
\hline 4 & Compactness Ratio & 2.76 \\
\hline 5. & Region Diameter & 1.37 \\
\hline 6. & Solidity Feature & 0.8 \\
\hline
\end{tabular}

TABLE 2. THRESHOLD SELECTION OF MORPHOLOGICAL FEATURES FOR ACCEPTABLE BROKEN GRAINS

\begin{tabular}{|c|c|c|}
\hline No. & Feature & Threshold Selection \\
\hline 1. & Average Length & 5.56 \\
\hline 2. & Aspect Ratio & 1.50 \\
\hline 3. & Compactness Ratio & 2.10 \\
\hline 4. & Region Diameter & 1.25 \\
\hline
\end{tabular}
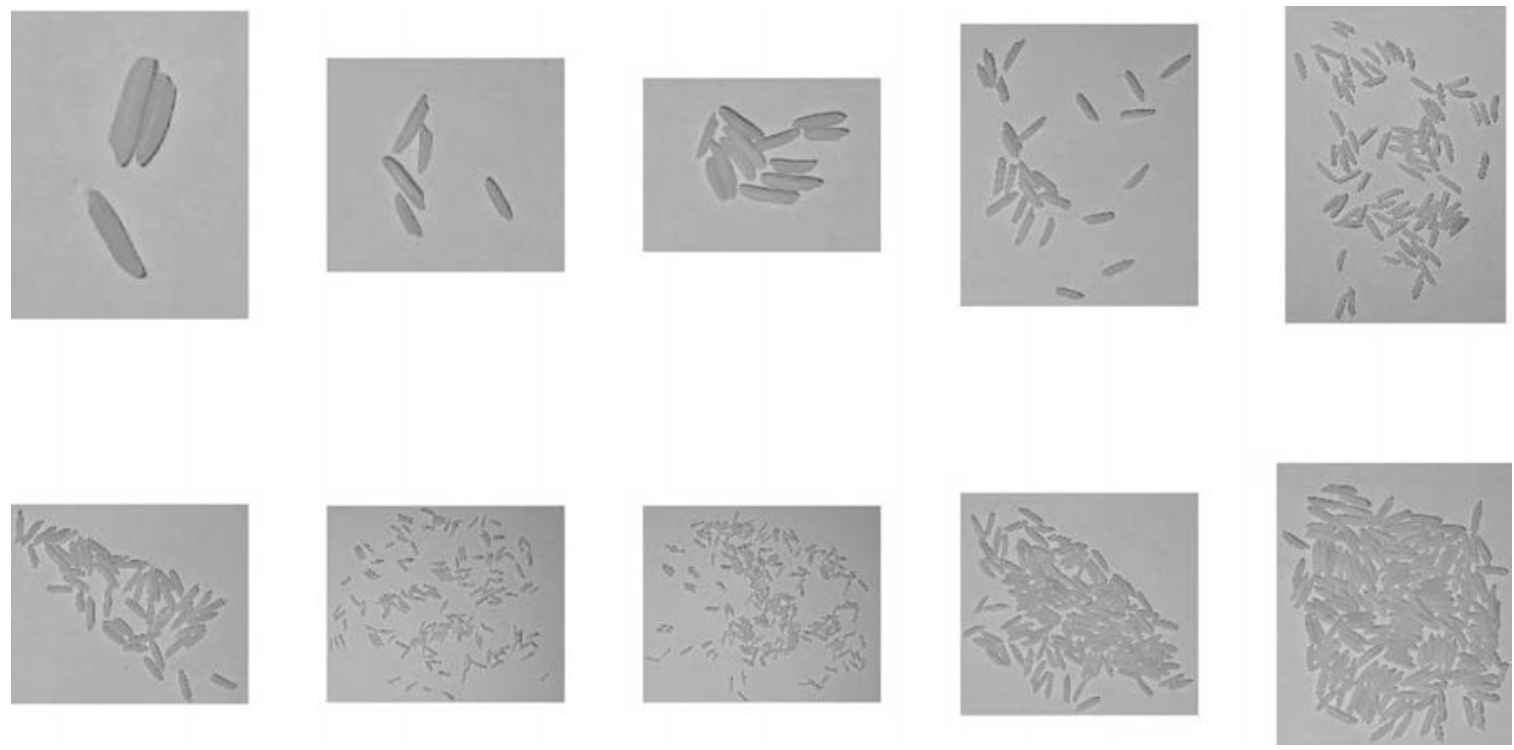

FIG. 2. TESTING IMAGES [34]

Mehran University Research Journal of Engineering \& Technology, Volume 35, No. 3, July, 2016 [p-ISSN: 0254-7821, e-ISSN: 2413-7219] 
Classification of Broken Rice Kernels using 12D Features

TABLE 3 PERFORMANCE RESULTS OF THE PROPOSED METHOD ON RICE KERNEL IMAGES

\begin{tabular}{|c|c|c|c|c|c|}
\hline Image & Specificity & Sensitivity & $\begin{array}{c}\text { Negative } \\
\text { Predicted Value }\end{array}$ & $\begin{array}{c}\text { Positive } \\
\text { Predicted Value }\end{array}$ & Accuracy \\
\hline 1. & 0.9852 & 0.6776 & 0.9522 & 0.8987 & 0.9635 \\
\hline 2. & 0.9869 & 0.6437 & 0.9574 & 0.8583 & 0.9562 \\
\hline 3. & 0.9454 & 0.8129 & 0.9769 & 0.6296 & 0.9418 \\
\hline 4. & 0.9917 & 0.5713 & 0.9406 & 0.9185 & 0.9483 \\
\hline 5. & 0.9894 & 0.6804 & 0.9526 & 0.8907 & 0.9443 \\
\hline 6. & 0.9837 & 0.6961 & 0.9552 & 0.8608 & 0.9509 \\
\hline 7. & 0.9878 & 0.6513 & 0.9441 & 0.8876 & 0.9305 \\
\hline 8. & 0.9726 & 0.7354 & 0.9707 & 0.7689 & 0.9457 \\
\hline 9. & 0.9826 & 0.7051 & 0.9564 & 0.8471 & 0.9495 \\
\hline 10. & 0.9906 & 0.5994 & 0.9406 & 0.9117 & 0.9612 \\
\hline Average & 0.9816 & 0.6773 & 0.9547 & 0.8472 & 0.9492 \\
\hline
\end{tabular}

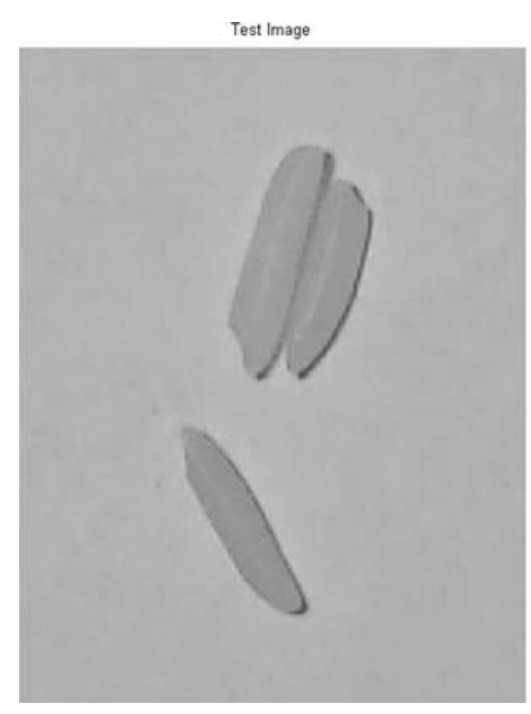

Results acquired by applying the proposed method

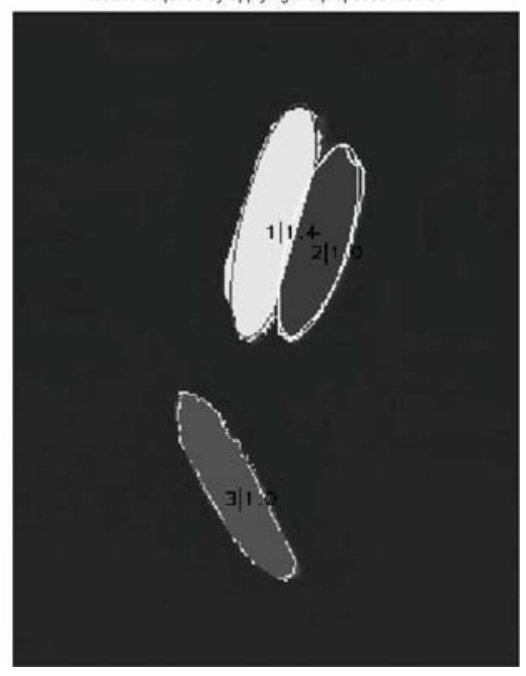

FIG. 3. THE RESULTS AFTER APPLYING THE PROPOSED METHOD
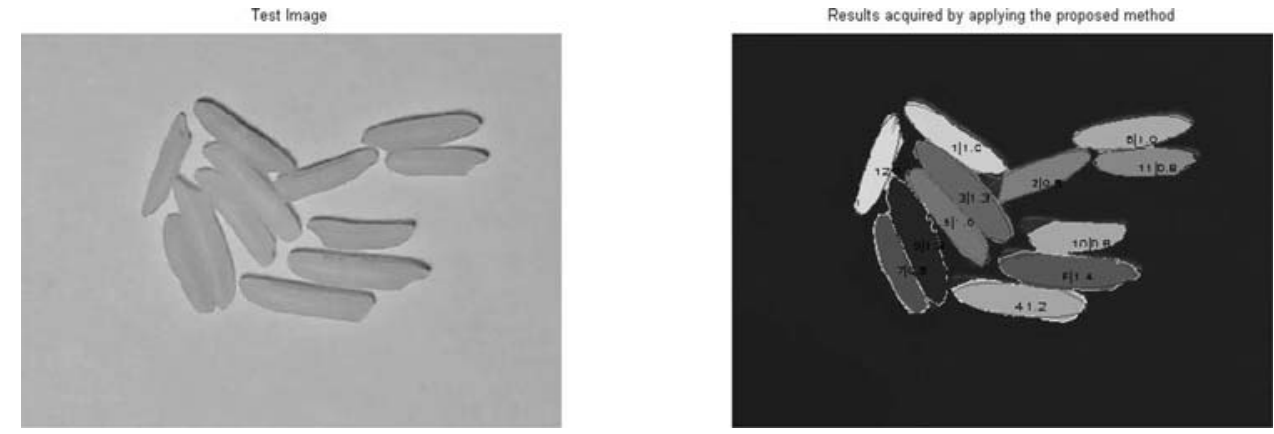

FIG. 4. RESULTS AFTER APPLYING THE PROPOSED METHOD

Mehran University Research Journal of Engineering \& Technology, Volume 35, No. 3, July, 2016 [p-ISSN: 0254-7821, e-ISSN: 2413-7219] 


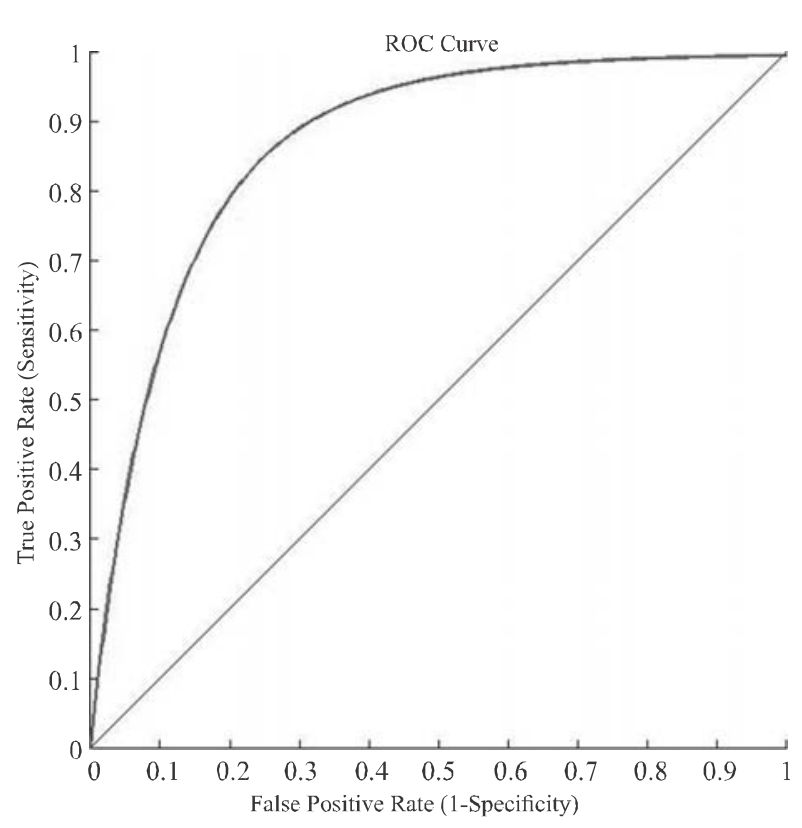

FIG. 5. ROC CURVE FOR MEASURED ACCURACY VALUES

\section{CONCLUSIONS}

This paper proposes a method based on 12D features which include morphological features, gray-level based features and Hough transform based feature to classify the rice kernels using RBF. Though these features are extensively used in the areas of image analysis and pattern recognition but these features haven't been used in conjunction with each other as proposed in this paper.

This paper focuses on two major problems for classifying the rice kernels. First problem refers to the traditional method of classification having only two classes' i.e. complete rice kernels and broken rice kernels. This problem was eliminated by applying the proposed features to extend the classes of classification, thus sorting the rice kernels in three categories. The rice kernels which were broken yet acceptable can be added to the class of broken but acceptable rice kernel. Second problem emphasize on the merging of rice kernels with either other rice kernels or with boundary hence creating complexity to correctly classify the number of complete rice kernels. The said problem was eliminated by using solidity feature and Hough transform based feature.
Experimental results prove that the proposed features deliver accurate results in the classification of rice grains. Results indicate that the proposed descriptors can yield desirable results for the classification of complete rice kernels, acceptable broken rice kernels and unacceptable broken rice kernels. It is also worth notifying that the proposed method uses only 12 features to classify the rice kernels efficiently hence needs a shorter computation time as compared to the existing algorithms which use larger feature vectors to classify the same. Since the implementation of the proposed method is based on simulation, it has the room for improvement with reference to the classification performance. The effectiveness of the proposed method makes it a suitable approach which can be integrated in industrial applications for automatic quality assessment of rice grains.

\section{ACKNOWLEDGEMENTS}

Authors would like to thank Dr. Rahmatullah, Institute of Research Promotion, for valuable information on rice kernels along with the scanners for determining the average length of the kernel. Authors are also indebted to the reviewers for the valuable comments and recommendations to improve the readability of the manuscript.

\section{REFERENCES}

[1] Abdullah, M.Z., Fathinul-Syahir, A.S., and Mohd-Azemi, B.M.N., "Automated Inspection System for Color and Shape Grading of Starfruit (Averrhoa Carambola L.) Using Machine Vision Sensor", Transactions of the Institute of Measurement and Control, Volume 27, No. 2, pp. 65-87, 2005.

[2] Louise, F., "Rice is Life", Journal of Food Composition and Analysis, Volume 18, No. 4, pp. 249-253, 2005.

[3] Kruthika, R., Muruganand, S., and Periasamy, A., "Matching of Different Rice Grains using Digital Image Processing", International Journal of Advanced Research in Electrical, Electronics and Instrumentation Engineering, Volume 2, No. 7, pp. 2937-2941, 2013. 
[4] Verma, B., "Image Processing Techniques for Grading and Classification of Rice", International Conference on Computer Communication and Technology, pp. 220-223, 2010

[5] Moreda, J.P., Ortiz-Canavate, J., Garcia-Ramos, F.J., and Ruiz-Altisent, M., "Non-Destructive Technology for Fruit and Vegetable Size Determination - A Review”, Elsevier Journal of Food Engineering, Volume 92, No. 2, pp. 119-136, 2009.

[6] Dalen, G.V., "Determination of the Size Distribution and Percentage of Broken Kernels of Rice Using Flatbed Scanning and Image Analysis", Elsevier Food Research International, Volume 37, No.1, pp. 51-58, 2004.

Yadav, B.K., and Jindal, V.K., "Modeling Changes in Milled Rice (Oryza Sativa L.) Kernel Dimensions during Soaking by Image Analysis", Elsevier Journal of Food Engineering, Volume 80, No. 1, pp. 359-369, 2007.

[8] Rad, S.J.M., Tab, F.A., and Mollazade, K., "Classification of Rice Varieties using Optimal Color and Texture Features and BP Neural Networks", $7^{\text {th }}$ Iranian Conference on Machine Vision and Image Processing, pp. 1-5, 2011.

[9] Ding, K., and Gunasekaran, S., "Shape Feature Extraction and Classification of Food Material using Computer Vision", Transactions of the American Society of Agriculture Engineers, Volume 37, No. 5, pp. 1537-1545, 1994.

[10] Uddin, M.Z., Lee, J.J., and Kim, T. S., “An Enhanced Independent Component Based Human Facial Expression Recognition from Video", IEEE Transactions on Consumer Electronics, Volume 55, No. 4, pp. 2216-2224, November, 2009.

[11] Schels, M., and Schwenker, F., "A Multiple Classifier system Approach for Facial Expressions in Image Sequences utilizing GMM Super Vectors", Proceedings of $20^{\text {th }}$ International Conference on Pattern Recognition, pp. 4251-4254, August, 2010.

[12] Kaur, H., and Singh, B., "Classification and Grading Rice using Muti-Class SVM", International Journal of Scientific and Research Publications, Volume 3, No. 4, pp. 1-5, 2013.
[13] Kumar, S., and Hebert, M., "Discriminative Fields for Modeling Spatial Dependencies in Natural Images”, Advances in Neural Information Processing Systems, Volume 16, MIT Press, Cambridge, USA, 2003.

[14] Liu, Z.Y., Cheng, F., Ying, Y.B., and Rao, X.Q., "Identification of Rice Seed Varieties using Neural Network", Journal of Zhejiang University Science-B, Volume 6, No. 11, pp. 1095-1100, 2005.

[15] Marin, D., Aquino, A., Gegundez-Arias, M.E., and Bravo, J.M., "A New Supervised method for Blood vessel Segmentation in Retinal Images by using Gray-Level and Moment-Invariants Based Features", IEEE Transactions on Medical Imaging, Volume 30, No. 1, pp. 146-158, 2010.

[16] Zhang, Q., Yeo, T.S., Tan, H.S., and Luo, Y., "Imaging of a Moving Target with Rotating Parts Based on the Hough Transform", IEEE Transactions on Geoscience and Remote Sensing, Volume 46, No. 1, pp. 291-299, 2008.

[17] Khowaja, S.A., Shah, S.M. Z.S., and Memon, M.U., "Noise Reduction Technique using Radial Basis Function Neural Networks", Volume 33, No. 3, pp. 278-285, 2014

[18] Bai, X., Zhou, F., and Jin, T., "Enhancement of Dim Small Target through Modified Top Hat Transformation under the Condition of Heavy Clutter", Elsevier Signal Processing, Volume 90, No. 5, pp. 1643-1654, 2010.

[19] Sapirstein, H.D., Neuman, M., Wright, E.H., Shwedyk, E., and Bushuk, W., “An Instrumental System for Cereal Grain Classification using Digital image Analysis", Elsevier Journal of Cereal Science, Volume 6, No. 1, pp. 3-14, 1987.

[20] Zayas, I., Pomeranz, Y., and Lai, F.S., "Discrimination of Wheat and Non-Wheat Components in Grain Samples by Image Analysis", Cereal Chemistry, Volume 66, No. 3, pp. 233-237, 1989.

[21] Zayas, I.Y., Martin, C.R., Steele, J.L., and Katsevich, A., "Wheat Classification using Image Analysis and CrushForce Parameters", Transactions of the American Society of Agriculture Engineers, Volume 39, No. 6, pp. 2199-2204, 1996. 
[22] Wee, C.Y., Paramesran, R., Takeda, F., Tsuzuki, T., Kadota, H., and Shimanouchi, S., "Classification of Rice Grains using Fuzzy ARTMAP Neural Network", Asia Pacific Conference on Circuits and Systems, Volume 2, pp. 223-226, 2002.

[23] Danying, W., Xiufu, Z., Zhiwei, Z., Neng, C., Jie, M., Qing, Y., Jianli, Y., and Xiyuan, L., "Correlation Analysis of Rice Grain Quality Characteristics", Volume 31, No. 8, pp. 1086-1091, 2005.

[24] Wee, C.Y., Paramesran, R., and Takeda, F., "Fast Computation of Zernike Moments for Rice Sorting Systems", IEEE International Conference on Image Processing, Volume 6, pp. 165-168, 2007.

[25] Agustin, O.C., and Byung-Joo, O., "Automatic Milled Rice Quality Analysis", $2^{\text {nd }}$ International Conference on Future Generation Communication and Networking, Volume 2, pp. 112-115, 2008.

[26] Pearson, T., "Hardware-Based Image Processing for High Speed Inspection of Grains", Elsevier's Computers and Electronics in Agriculture, Volume 69, No. 1, pp. 12-18, 2009.

[27] Pabamalie, L.A.I., and Premaratne, H.L., "A Grain Quality Classification System”, International Conference on Information Society, pp. 56-61, 2010.

[28] Rad, S.J.M., Tab, F.A., and Mollazade, K., "Design of an Expert System for Rice Kernel Identification using Optimal Morphological Features and Back Propagation Neural Network", International Journal of Applied Information Systems, Volume 3, No. 2, pp. 33-37, 2012.

[29] Pazoki, A.R., Farokhi, F., and Pazoki, Z., "Classification of Rice Grain Varieties using Two Artificial Neural Networks (MLP and Neuro-Fuzzy)", The Journal of Animal and Plant Sciences, Volume 24, No. 1, pp. 336-343, 2014.

[30] Xu, S., Zhou, Z., Lu, H., Luo. X., and Lan, Y., "Improved Algorithms for the Classification of Rough Rice using a Bionic Electronic Nose Based on PCA and the Wilks Distribution", Sensors, Volume 14, No. 3, pp. 5486-5501, 2014.
[31]

Abdullah, A.H., Adom, A.H., Shakaff, A.Y.M., Masnan, M.J., Zakaria, A., Rahim, N.A., and Omar, O., "Classification of Malaysia Aromatic Rice using Multivariate Statistical Analysis", International Conference on Mathematical Engineering and Industrial Applications, Volume 1660, pp. 090005, 2015.

[32] Wang, L., Liu, D., Pu, H., Sun, D.W., Gao, W., and Xiong, Z., "Use of Hyperspectral Imaging to Discriminate the Variety and Quality of Rice", Food Analytical Methods, Volume 8, No. 2, pp. 515-523, 2015.

[33] Zhang, B., Wu, X., You, J., Li, Q., and Karray, F., "Detection of Microaneurysms using Multi-Scale Correlation Coefficients", Elsevier Pattern Recognition Letters, Volume 43, No. 6, pp. 2237-2248, June, 2010.

[34] FTP Site for Counting Grains of Rice http:// codegolf.stackexchange.com/questions/40831/countinggrains-of-rice", Date accessed: 29/09/2015.

[35] Ngampak, D., and Piamsanga, P., "Image Analysis of Broken Rice Grains of Khao Dawk Mali Rice", $7^{\text {th }}$ International Conference on Knowledge and Smart Technology, pp. 115-120, January, 2015.

[36] Neimeijer, M., Ginneken, V.B., Staal, J.J., SuttorpSchulten, M.S.A., and Abramoff, M.D., "Automatic Detection of Red Lesions in Digital Color Fundus Photographs", IEEE Transactions on Medical Imaging, Volume 24, No. 5, pp. 584-592, May, 2005.

[37] Zhang, B., Wu, X., You, J., Li, Q., and Karray, F., "Detection of Microaneurysms using Multi-Scale Correlation Coefficients", Pattern Recognition Letters, Volume 43, pp. 2237-2248, 2010.

[38] Jagoe, R., Arnold, J., Blauth, C., Smith, P.L.C., Taylor, K.M., and Wootton, R., "Measurement of Capillary Dropout in Retinal Angiograms by Computerized Image Analysis", Pattern Recognition Letters, Volume 13, pp. 143-151, 1992. 